\title{
Análisis de los Flujos de Potencia de Sistemas Eléctricos y su Interpretación Física
}

\author{
Nicolás Muñoz, Fernando Villada y Diego R. Cadavid \\ Universidad de Antioquia, Fac. de Ingeniería, Depto. Ing. Eléctrica, Grupo de Manejo Eficiente de \\ la Energía - GIMEL, Calle 67 No. 53-108, Oficina 19-437, Medellín-Colombia \\ (e-mail:nicolasm@udea.edu.co, fvillada@udea.edu.co,dcadavid@udea.edu.co)
}

Recibido Mar. 19, 2012; Aceptado Abr. 27, 2012; Versión final recibida May. 14, 2012

\section{Resumen}

En este artículo se extiende la teoría de la potencia instantánea del Instituto de Ingenieros Eléctricos y Electrónicos (IEEE, Std. 1459 de 2010) usada en sistemas eléctricos monofásicos a sistemas eléctricos trifásicos. A partir del producto de voltajes y corrientes instantáneos se obtiene una expresión general de potencia instantánea que sirve para explicar los fenómenos de los sistemas eléctricos. Esta expresión se verifica mediante pruebas experimentales relacionando los flujos de potencia con las causas que lo producen. Existen numerosas expresiones de potencia instantánea reportadas por la literatura pero ninguna de ellas relaciona físicamente ni matemáticamente los fenómenos de los sistemas eléctricos con las causas que lo producen. Por ello se propone el uso de la teoría de la potencia instantánea para explicar los fenómenos de los sistemas eléctricos y contribuir con una teoría de potencia que simultáneamente pueda facturar energía, evaluar la calidad de la energía, diseñar filtros activos, y detectar las fuentes de distorsión armónica.

\section{Analysis and Physical Interpretation of Electric System Power Flow}

\begin{abstract}
In this paper, the instantaneous power theory of the Institute of Electrical and Electronics Engineers (IEEE, Std. 1459 of 2010) used for single-phase electric systems is expanded to threephase electric systems. A general instantaneous power expression is obtained through instantaneous voltages and currents. This expression is useful to explain the phenomena in electrical systems. This expression is also verified through experimental tests relating power flow and its causes. There are numerous instantaneous power expression reported in the literature, but these expressions do not relate physically and mathematically the electrical phenomena and its causes. For this reason, it is proposed to use the instantaneous power theory to explain physical phenomena in electrical systems contributing with a power theory that can be simultaneity used for measuring or monitoring to revenue purposes, for evaluating the power quality, for designing active filters, and for detecting sources of harmonic distortion.
\end{abstract}

Keywords: instantaneous power theory, power flows, active power, reactive power 


\section{INTRODUCCIÓN}

Desde hace más de un siglo, numerosos científicos se han preocupado por intentar explicar los fenómenos existentes en los sistemas eléctricos. Sus trabajos han dado lugar a la aparición, a lo largo del tiempo, de las diferentes teorías de la potencia eléctrica existentes. Una teoría de la potencia eléctrica debe entenderse como un conocimiento desde el nivel más básico de las propiedades de los sistemas eléctricos y a su vez debe establecer las herramientas matemáticas que proporcionen una interpretación física del conjunto de fenómenos propios de los sistemas eléctricos. Las teorías de la potencia instantánea surgen a partir de desarrollos matemáticos complejos, con mayor o menor grado de abstracción, en los que no se puede establecer una relación directa entre las magnitudes calculadas y los fenómenos físicos reales presentes en los sistemas eléctricos. Al no existir una relación directa entre las magnitudes utilizadas y los fenómenos físicos reconocidos en los sistemas eléctricos (desfase, desequilibrio y distorsión), se pueden cometer errores en la cuantificación y medición de la potencia en los sistemas eléctricos.

La IEEE Std. 1459 (2010) fue elaborada con el objetivo de ser base para la nueva generación de equipos de medición para la potencia eléctrica, Pigazo y Moreno (2007). La IEEE Std 1459 (2010), presenta la teoría de la potencia sólo para sistemas monofásicos, en este artículo esta teoría es extendida para sistemas trifásicos explicando el significado físico de los flujos de potencia. Emmanuel (2004) presenta un resumen de las principales definiciones de la IEEE Std. 1459 (2010), mientras que Willems et al. (2005) explican el concepto de potencia aparente de la IEEE Std. 1459 (2010) para diferentes definiciones de voltaje y corriente efectivos.

Existen numerosos trabajos en los que se utilizan los flujos de potencia, algunos de estos trabajos son citados a continuación: Chen y Hsu (2000) utilizan los flujos de potencia para analizar sistemas de distribución de cuatro hilos con tensiones asimétricas distorsionadas y con carga desequilibrada usando la transformada de Fourier y la transformada de Fortesque, este trabajo no relaciona los fenómenos físicos de los sistemas eléctricos con las magnitudes que los cuantifican. Emanuel (1993a) utiliza los flujos de potencia para analizar sistemas eléctricos desequilibrados con tensiones y corrientes senosoidales, la suma de las amplitudes de los flujos de potencia en cada fase se utilizan para cuantificar los flujos de potencia reactivos oscilantes. Emmanuel (1990) explica el significado físico para la potencia reactiva cuando existen tensiones y corrientes distorsionadas para sistemas eléctricos monofásicos. Emanuel (1993b) presenta una expresión de potencia instantánea particularizándola sólo para la fase $b$. Ferrero y Superti (1991) presentan la descomposición de las potencias activas y no activas mediante la teoría de Park. Akagi et al. (1999), y Kim et al. (2002) explican y analizan la descomposición de la potencia en componentes ortogonales. Salmeron y Montaño (1996) mediante la teoría de la potencia eléctrica generalizada relacionan físicamente cada corriente con cada término de potencia que provoca. Czarnecki (1988) realiza una descomposición de corriente asociándolas con los fenómenos de los sistemas eléctricos. Depenbrock (1993) relaciona voltajes, corrientes, y potencias instantáneas mediante el método FBD.

La mayoría de los trabajos consultados relacionan los fenómenos de los sistemas eléctricos con las corrientes que los provocan y discriminan entre la potencia útil y la no útil de los sistemas eléctricos deduciendo que la potencia activa representa la transferencia útil de potencia. En este artículo se demuestra lo que es enunciado en la IEEE Std. 1459 (2010), que $\mathrm{P}_{1}^{+}$cuantifica el flujo de potencia útil de los sistemas eléctricos. En el artículo también se establece matemática y físicamente la relación directa entre los flujos de potencia y las causan que lo producen para los sistemas trifásicos tal y como lo realiza la IEEE Std. 1459 (2010) para los sistemas monofasicos.

El artículo básicamente extiende la teoría de la potencia instantánea de la IEEE Std. 1459 (2010) para sistemas eléctricos monofásicos a sistemas eléctricos trifásicos. Se propone esta teoría para explicar los fenómenos físicos que surgen en los sistemas eléctricos trifásicos y contribuir con una teoría que simultáneamente pueda cuantificar el intercambio de potencia entre empresas de distribución de energía y los usuarios o clientes. Así como evaluar la calidad de la potencia, y establecer estrategias de compensación de potencia ineficiente. 


\section{LA TEORÍA DE LA POTENCIA INSTANTÁNEA DE LA IEEE STD. 1459}

En la IEEE Std. 1459 (2010), se usa la teoría de la potencia instantánea para sistemas eléctricos monofásicos. Para sistemas monofásicos lineales, es decir para sistemas alimentados con una tensión que sólo contiene la componente fundamental y que sólo demanda la componente fundamental de corriente, el grupo de trabajo del estándar define la potencia instantánea fundamental $\left(\mathrm{p}_{1}\right.$, ecuación 1) conformada por la potencia activa instantánea fundamental $\left(\mathrm{P}_{\mathrm{a} 1}\right)$ y la potencia reactiva instantánea fundamental $\left(\mathrm{P}_{\mathrm{q} 1}\right)$ :

$$
\mathrm{p}_{1}=\mathrm{p}_{\mathrm{a} 1}+\mathrm{p}_{\mathrm{q} 1}=\left[\mathrm{V}_{1} I_{1} \cos \theta_{1}\left[1-\cos \left(2 \omega_{1} \mathrm{t}\right)\right]\right\}+\left[-\mathrm{V}_{1} \mathrm{I}_{1} \operatorname{sen} \theta_{1} \operatorname{sen}\left(2 \omega_{1} \mathrm{t}\right)\right\}
$$

Donde: $V_{1}$ e $I_{1}$ son la amplitud de la tensión aplicada y de la corriente demandada por la carga, $\theta_{1}$ es el desfase fundamental que existe entre la tensión y la corriente, y $\omega_{1}$ es la frecuencia angular fundamental. El valor medio de $\mathrm{P}_{\mathrm{a} 1}(\mathrm{t})$ corresponde a la potencia activa fundamental $\left(\mathrm{P}_{1}\right.$, ecuación 2). El flujo $\mathrm{Pa}_{\mathrm{a}}(\mathrm{t})$ se considera eficiente ya que su valor medio es positivo. Esto significa que el flujo $\mathrm{p}_{\mathrm{a} 1}(\mathrm{t})$ es unidireccional y fluye desde la red de suministro hacia la carga.

$P_{1}=\frac{1}{k T} \int_{\tau}^{\tau+k T} p_{a 1} d t=V_{1} I_{1} \cos \theta_{1}$

El valor medio de $\mathrm{p}_{\mathrm{q} 1}$ es nulo, esto significa que existe un flujo bidireccional (ineficiente) de potencia entre la red de suministro y la carga, por lo que el intercambio de potencia es cero. Debido a que el intercambio de potencia es cero, el flujo de $\mathrm{P}_{\mathrm{q} 1}$ es considerado ineficiente y sólo ocasiona pérdidas de potencia cuando fluye por el sistema eléctrico, para cuantificar el flujo de $\mathrm{p}_{\mathrm{q} 1}$ utiliza su amplitud ( $\mathrm{Q}_{1}$, ecuación (3)).

$Q_{1}=V_{1} I_{1} \operatorname{sen} \theta_{1}$

Para sistemas eléctricos monofásicos no lineales, la IEEE Std. 1459 (2010) muestra una expresión de potencia instantánea ( $\mathrm{p}$, ecuación (4)) que se compone de la potencia activa instantánea $\left(\mathrm{P}_{\mathrm{a}}\right)$ y potencia reactiva instantánea $\left(\mathrm{P}_{\mathrm{q}}\right)$ :

$$
\begin{aligned}
& \mathrm{p}=\mathrm{p}_{\mathrm{a}}+\mathrm{p}_{\mathrm{q}} \\
& \mathrm{p}_{\mathrm{a}}=\sum_{h=1}^{\infty} \mathrm{V}_{\mathrm{h}} \mathrm{I}_{\mathrm{h}} \cos \theta_{\mathrm{h}}\left[1-\cos \left(2 \mathrm{~h} \omega_{1} \mathrm{t}\right)\right] \\
& \mathrm{p}_{\mathrm{q}}=\sum_{\mathrm{h}=1}^{\infty} \mathrm{V}_{\mathrm{h}} \mathrm{I}_{\mathrm{h}} \operatorname{sen} \theta_{\mathrm{h}} \operatorname{sen}\left(2 \mathrm{~h} \omega_{1} \mathrm{t}\right)+\sum_{\substack{\mathrm{m} \neq \mathrm{n} \\
\mathrm{m}, \mathrm{n} \neq 1}}^{\infty} 2 \mathrm{~V}_{\mathrm{m}} \mathrm{I}_{\mathrm{n}} \operatorname{sen}\left(\mathrm{m} \omega_{1} \mathrm{t}+\alpha_{\mathrm{m}}\right) \operatorname{sen}\left(\mathrm{n} \omega_{1} \mathrm{t}+\beta_{\mathrm{n}}\right)
\end{aligned}
$$

$p_{a}$ contiene términos de potencia instantánea fundamentales y no fundamentales. El valor medio de $\mathrm{P}_{\mathrm{a}}$ es positivo y contiene a $\mathrm{P}_{1}$ y a la potencia activa armónica $\left(\mathrm{P}_{\mathrm{H}}\right.$, ecuación (7)). A pesar de que $\mathrm{P}_{\mathrm{H}}$ corresponde a un flujo unidireccional de la red de suministro a la carga (valor medio positivo), es considerado ineficiente, ya que provoca pérdidas de potencia en los conductores, es muy perjudicial para las cargas electrónicas sensibles, y ocasiona problemas de estabilidad en motores y generadores, Giadrossi et al. (2008). Al igual que $\mathrm{Pa}_{\mathrm{a}}$ la potencia $\mathrm{P}_{\mathrm{q}}$ contiene términos de potencia instantánea fundamentales y no fundamentales. El valor medio de $\mathrm{Pq}_{\mathrm{q}}$ es cero, por lo que el flujo de potencia es bidireccional y no representa una transferencia neta de potencia a la carga, por esta razón es considerado ineficiente. Para cuantificar el flujo de potencia de $\mathrm{p}_{\mathrm{q}}$ se utiliza la expresión de la ecuación (8): 


$$
\begin{aligned}
& \mathrm{P}_{\mathrm{H}}=\sum_{h \neq 1}^{\infty} \mathrm{V}_{h} \mathrm{I}_{h} \cos \theta_{\mathrm{h}} \\
& \mathrm{Q}=\mathrm{Q}_{1}+\mathrm{Q}_{\mathrm{H}}=\sum_{h=1}^{\infty} \mathrm{V}_{\mathrm{h}} I_{\mathrm{h}} \operatorname{sen} \theta_{\mathrm{h}}
\end{aligned}
$$

\section{TEORIA DE LA POTENCIA INSTANTÁNEA EXTENDIDA A SISTEMAS TRIFÁSICOS}

En esta sección se extenderá la teoría de la potencia instantánea de la IEEE Std. 1459 (2010) usada en sistemas eléctricos monofásicos a sistemas eléctricos trifásicos. Generalmente al multiplicar voltajes y corrientes instantáneos en las ecuaciones de potencia aparecen términos cuadráticos sinusoidales, con los que no es posible explicar el flujo de potencia del sistema ni mucho menos identificar que flujos son eficientes o ineficientes. En esta sección se obtendrán expresiones de potencia instantánea a partir de la descomposición de sus términos cuadráticos en términos sinusoidales puros. Estas nuevas expresiones de potencia instantánea tienen interpretación física y con ellas es posible realizar la cuantificación de la potencia eléctrica.

Se propondrán expresiones de potencia instantánea para sistemas lineales, no lineales, equilibrados y desequilibrados reflejando lo que sucede en cada instante de tiempo en el sistema de potencia, lo que las convierte en expresiones valiosas que pueden ser usadas en filtros y compensadores activos, Orts et al. (2010).

Potencia instantánea para sistemas trifásicos lineales y equilibrados.

En este caso debido a que el sistema es lineal y equilibrado sólo existen componentes fundamentales de voltaje de secuencia positiva ( $\left.V_{\mathrm{a} 1}=V_{b 1}=V_{c 1}=V_{1}^{+}\right)$y de corriente de secuencia positiva $\left(\mathrm{I}_{\mathrm{a} 1}=\mathrm{I}_{b 1}=\mathrm{I}_{\mathrm{c} 1}=I_{1}^{+}\right)$. Se define la potencia instantánea fundamental de secuencia positiva $\left(\mathrm{P}_{z 1}^{+}\right)$en función del voltaje $\left(\mathrm{v}_{z 1}^{+}=\sqrt{2} \mathrm{~V}_{1}^{+} \operatorname{sen}\left(\omega_{1} t-\varphi_{\mathrm{z}}\right)\right.$ ), y corriente $\left(\mathrm{i}_{\mathrm{z} 1}^{+}=\sqrt{2} \mathrm{I}_{1}^{+} \operatorname{sen}\left(\omega_{1} t-\varphi_{\mathrm{z}}+\beta_{1}^{+}\right)\right.$) instantáneas fundamentales de secuencia positiva:

$\mathrm{p}_{z 1}^{+}=\mathrm{v}_{z 1}^{+} \mathrm{i}_{z 1}^{+}=2 \mathrm{~V}_{1}^{+} I_{1}^{+} \operatorname{sen}\left(\omega_{1} \mathrm{t}-\varphi_{z}\right) \operatorname{sen}\left(\omega_{1} \mathrm{t}-\varphi_{\mathrm{z}}+\beta_{1}^{+}\right)$

Donde, $\varphi_{z}$ es la fase inicial de los voltajes línea neutro $\left(\varphi_{\mathrm{a}}=0, \varphi_{\mathrm{b}}=2 \pi / 3, \varphi_{\mathrm{c}}=4 \pi / 3\right)$, $\beta_{1}^{+}$es el desfase que existe entre los voltajes línea neutro y las corrientes de las fases. $\mathrm{p}_{\mathrm{z} 1}^{+}$puede transformarse en términos de funciones sinusoidales simples y expresarse en términos de la potencia instantánea activa $\left(\mathrm{p}_{z 1 \mathrm{p}}^{+}\right)$y reactiva $\left(\mathrm{P}_{z 1 \mathrm{q}}^{+}\right)$por fase como sigue:

$\mathrm{p}_{z 1}^{+}=\mathrm{p}_{\mathrm{z} 1 \mathrm{p}}^{+}+p_{\mathrm{z} 1 \mathrm{q}}^{+}=\mathrm{V}_{1}^{+} I_{1}^{+} \cos \theta_{1}^{+}\left[1-\cos \left(2 \omega_{1} \mathrm{t}-2 \varphi_{\mathrm{z}}\right)\right]-\mathrm{V}_{1}^{+} \mathrm{I}_{1}^{+} \operatorname{sen} \theta_{1}^{+} \operatorname{sen}\left(2 \omega_{1} \mathrm{t}-2 \varphi_{\mathrm{z}}\right)$

La potencia instantánea trifásica $\left(\mathrm{p}_{1}^{+}\right)$puede obtenerse mediante la suma de las potencias instantáneas activas por fase y puede expresarse en función de la potencia instantánea activa trifásica $\left(\mathrm{p}_{1 \mathrm{p}}^{+}\right)$y potencia instantánea reactiva trifásica $\left(\mathrm{p}_{1 \mathrm{q}}^{+}\right)$:

$$
\begin{aligned}
& \mathrm{p}_{1}^{+}=\sum_{\mathrm{z}=\mathrm{a}, \mathrm{b}_{i} \mathrm{c}} \mathrm{p}_{\mathrm{z} 1}^{+}=p_{1 \mathrm{p}}^{+}+\mathrm{p}_{1 \mathrm{q}}^{+} \\
& \mathrm{p}_{1 \mathrm{p}}^{+}=\mathrm{V}_{1}^{+} \mathrm{I}_{1}^{+} \cos \theta_{1}^{+}\left[\left[1-\cos 2 \omega_{1} \mathrm{t}\right]+\left[1-\cos \left(2 \omega_{1} \mathrm{t}-\frac{4 \pi}{3}\right)\right]+\left[1-\cos \left(2 \omega_{1} \mathrm{t}-\frac{2 \pi}{3}\right)\right]=3 \mathrm{~V}_{1}^{+} I_{1}^{+} \cos \theta_{1}^{+}\right. \\
& \mathrm{p}_{1 \mathrm{q}}^{+}=-\mathrm{V}_{1}^{+} \mathrm{I}_{1}^{+} \operatorname{sen} \theta_{1}^{+}\left[\operatorname{sen} 2 \omega_{1} \mathrm{t}+\operatorname{sen}\left(2 \omega_{1} \mathrm{t}-\frac{4 \pi}{3}\right)+\operatorname{sen}\left(2 \omega_{1} \mathrm{t}-\frac{2 \pi}{3}\right)\right]=0
\end{aligned}
$$

$\mathrm{p}_{1 p}^{+}$es una función sinusoidal de valor siempre positivo, por lo que representa un flujo 
unidireccional de potencia desde la red de suministro hacia la carga. $\mathrm{p}_{1 \mathrm{q}}^{+}$es una función sinusoidal de valor medio nulo, por lo que representa un flujo bidireccional (ineficiente) entre la red de suministro y la carga. En cuanto a $\mathrm{p}_{1}^{+}$es un valor constante, ya que se cancelan entre sí los términos cosenoidales dependientes del tiempo y su valor es el triple de la potencia instantánea activa de cualquiera de las fases del sistema trifásico equilibrado $\left(\mathrm{p}_{z 1}^{+}\right)$. La potencia instantánea reactiva trifásica $\left(\mathrm{P}_{1 \mathrm{q}}\right)$, está formada por la suma de las potencias instantáneas reactivas por fase, y debido a que se trata de un sistema de tensiones senoidales de valor medio igual a cero y desfasadas $2 \pi / 3$ entre si, que al sumarse entre ellas dan igual a cero en cualquier instante. De esta manera, $\mathrm{p}_{1}$ en este caso coincide con $\mathrm{p}_{1 \mathrm{p}}$ y la expresión de potencia reactiva $\mathrm{p}_{1 \mathrm{q}}$ no se ve reflejada en la expresión de potencia instantánea trifásica. Así que, la potencia instantánea en un sistema trifásico equilibrado y lineal es constante e igual a tres veces la potencia activa de una de las fases. $\mathrm{p}_{1}$ es un valor constante en el tiempo de valor medio diferente a cero, por lo tanto, es la potencia útil que es entregada de la red de suministro a la carga coincidiendo con la potencia activa del sistema. Además, de acuerdo con las características del voltaje y la corriente, la potencia activa del sistema eléctrico en este caso, es la potencia activa fundamental de secuencia positiva $\left(\mathrm{P}_{1}^{+}\right)$y puede definirse como sigue:

$\mathrm{P}_{1 \mathrm{p}}^{+}==3 \mathrm{~V}_{1}^{+} \mathrm{I}_{1}^{+} \cos \theta_{1}^{+}$

La expresión (14) coincide con la expresión de potencia activa definida por la IEEE Std. 1459 (2010). De acuerdo con el análisis realizado, la potencia reactiva trifásica corresponde con la potencia reactiva fundamental de secuencia positiva ( $\left.Q_{1}^{+}\right)$de la IEEE Std. 1459 (2010) y se puede definir a partir de la suma de las amplitudes de las potencias instantáneas bidireccionales de cada fase:

$Q_{1}^{+}=Q_{a}^{+}+Q_{b}^{+}+Q_{c}^{+}=3 V_{1}^{+} I_{1}^{+} \operatorname{sen} \theta_{1}^{+}$

La potencia aparente trifásica corresponde con la potencia aparente de secuencia positiva $\left(\mathrm{S}_{1}^{+}\right)$de la IEEE Std. 1459 (2010) y está dada por:

$\left(\mathrm{S}_{1}^{+}\right)^{2}=\left(\mathrm{P}_{1}^{+}\right)^{2}+\left(\mathrm{Q}_{1}^{+}\right)^{2}=\left(3 \mathrm{~V}_{1}^{+} I_{1}^{+}\right)^{2}$

Potencia instantánea para sistemas trifásicos lineales y desequilibrados.

En este caso, se consideran voltajes senoidales y asimétricos de frecuencia fundamental $\left(V_{\mathrm{a} 1} \neq V_{b 1} \neq V_{\mathrm{c} 1}\right)$ que pueden expresarse en términos de las componentes simétricas $\left(V_{1}^{+}, V_{1}^{-}, y\right.$ $\left.V_{1}^{0}\right)$, el voltaje instantáneo por fase $\left(v_{z 1}=v_{z 1}^{+}+v_{z 1}^{{ }_{z} v_{L_{1}}^{Q}}\right)$ es:

$v_{z 1}=\sqrt{2} V_{1}^{+} \operatorname{sen}\left(\omega_{1} t-\varphi_{z}\right)+\sqrt{2} V_{1}^{-} \operatorname{sen}\left(\omega_{1} t+\varphi_{z}\right)+\sqrt{2} V_{1}^{0} \operatorname{sen}\left(\omega_{1} t\right)$

Donde, $\varphi_{z}$ es la fase inicial de los voltajes línea neutro $\left(\varphi_{\mathrm{a}}=0, \varphi_{\mathrm{b}}=2 \pi / 3, \varphi_{\mathrm{c}}=4 \pi / 3\right)$, las corrientes son senoidales y desequilibradas de frecuencia fundamental $\left(I_{a 1} \neq I_{b 1} \neq I_{c 1}\right)$, y pueden expresarse en términos de las componentes simétricas $\left(\mathrm{I}_{1}^{+}, \mathrm{I}_{1}^{-}, \mathrm{e} \mathrm{I}_{1}^{0}\right)$, la corriente instantánea por fase $\left(i_{z 1}^{i}=i_{z 1}^{+}+i_{z 1}^{\bar{F}_{21} i_{1}}\right.$ ) es:

$\mathrm{i}_{z 1}=\sqrt{2} \mathrm{I}_{z 1}^{+} \operatorname{sen}\left(\omega_{1} t-\varphi_{z}+\beta_{z 1}^{+}\right)+\sqrt{2} I_{z 1}^{-} \operatorname{sen}\left(\omega_{1} t+\varphi_{z+} \beta_{z 1}^{-}\right)+\sqrt{2} I_{z 1}^{0} \operatorname{sen}\left(\omega_{1} t+\beta_{z 1}^{0}\right)$

La potencia instantánea por fase es el producto entre el voltaje instantáneo por fase $\left(\mathrm{v}_{z 1}\right)$ y la corriente instantánea por fase $\left({ }^{\pi} \mathbb{i}_{z 1}\right)$ :

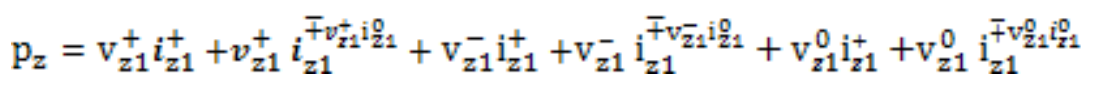


El primer término de (19) corresponde al producto del voltaje instantáneo de secuencia positiva y la corriente instantánea de secuencia positiva y su análisis es idéntico al análisis de la sección anterior. El resto de términos corresponden a flujos de potencia que aparecen debido a la asimetría de la fuente de tensión y al desequilibrio de la carga siendo ineficientes porque son flujos bidireccionales o causan pérdidas de potencia o problemas en los sistemas eléctricos. A continuación, a modo de ejemplo, será desarrollada la segunda expresión de ecuación (19), al estar formada por un voltaje de secuencia positiva $\left(v_{z 1}^{+}=\sqrt{2} V_{1}^{+} \operatorname{sen}\left(\omega_{1} t-\varphi_{z}\right)\right.$ y una corriente de secuencia negativa $\left(i_{z 1}^{-}=\sqrt{2} I_{1}^{-} \operatorname{sen}\left(\omega_{1} t+\varphi_{z}+\beta_{z 1}^{-}\right)\right)$puede ser reducida como sigue:

$\mathrm{p}_{z}^{\ddagger}=V_{1}^{+} I_{1}^{-} \cos \left(\theta_{1_{z}^{+} \mathrm{n} \varphi}\right)-\mathrm{V}_{1}^{+} \mathrm{I}_{1}^{-} \cos \left(2 \omega_{1} \mathrm{t}+\theta_{1}^{-}\right)$

La expresión reducida de la derecha contiene dos componentes de potencia instantáneas por fase de valor medio no nulo. La potencia instantánea trifásica provocada por corrientes de secuencia negativa $\left(\mathrm{p}^{ \pm}\right)$se puede obtener si las potencias instantáneas por fases son sumadas:

$\mathrm{p}^{+-}(\mathrm{t})=\mathrm{V}_{1}^{+} I_{1}^{-}\left\{\cos \left(\theta_{1}^{-}\right)+\cos \left(\theta_{1}^{-}+4 \pi / 3\right)+\cos \left(\theta_{1}^{-}+2 \pi / 3\right)-3 \cos \left(2 \omega_{1} t+\theta_{1}^{-}\right)\right\}$

$=-3 V_{1}^{+} I_{1}^{-} \cos \left(2 \omega_{1} t+\theta_{1}^{-}\right)$

Su valor medio es nulo, por lo que, al igual que ocurría con la potencia reactiva instantánea, representa un flujo bidireccional (ineficiente) de energía que no se transforma en potencia útil y por lo tanto representa una ineficiencia del sistema.

Es preciso notar que a pesar de que existe potencia por fase cuyo valor medio es no nulo (potencia eficiente), este tipo de energía finalmente es ineficiente ya que desde el punto de vista trifásico, las potencias instantáneas de todas las fases se compensan o se anulan en la carga y no hay transferencia útil de energía desde la red de suministro hacia la carga, sólo existe un intercambio de energía producido por una potencia instantánea bidireccional (ineficiente).

Aplicando la teoría de la potencia instantánea, se propone la cuantificación de la potencia debido a la presencia de corrientes fundamentales de secuencia negativa, al igual que se ha hecho con la potencia debido a los desfases, como la suma de las amplitudes de la potencia instantánea de cada una de las fases $\left(A_{i}\right)\left(A_{i}\right.$ coincide con la potencia de desequilibrio de la IEEE Std 1459 (2010) $\left(\mathrm{S}_{\mathrm{U} 1}\right)$ cuando sólo existen $\left.\mathrm{V}_{1}^{+} \mathrm{e}, \mathrm{I}_{1}^{-}\right)$:

$\mathrm{A}_{\mathrm{i}}=\sum_{\mathrm{z}=\mathrm{a}, \mathrm{b}, \mathrm{c}} \max \left|\mathrm{p}_{\mathrm{z}}^{ \pm}\right|=3 \mathrm{~V}_{1}^{+} \mathrm{I}_{1}^{-}$

Potencia instantánea para sistemas trifásicos no lineales.

En este caso se encontrará una expresión general para la potencia instantánea que contempla cargas no lineales y tensiones no sinusoidales. Para lograrlo, se requiere usar expresiones de voltaje y corriente instantáneos de frecuencias y fases diferentes. Para un sistema trifásico con armónicos de corriente y de tensión, la potencia instantánea por fase $\left(\mathrm{p}_{z}\right)$ puede escribirse en función del voltaje y corriente instantáneos como sigue:

$$
\begin{aligned}
& p_{z}(t)=\sqrt{2} \sum_{m=1}^{\infty} V_{z m} \operatorname{sen}\left(m\left(\omega_{1} t-\varphi_{z}\right)+\alpha_{z m}\right) \cdot \sqrt{2} \\
& \sum_{1=1}^{\infty} l_{z 1} \operatorname{sen}\left(n\left(\omega_{1} t-\varphi_{z}\right)+\beta_{z 1}\right)
\end{aligned}
$$

El subíndice $\mathrm{m}$, en este caso indica el orden del armónico de tensión y es usado para diferenciar los armónicos de tensión con los armónicos de corriente de subíndice $\mathrm{n}$. El desarrollo de las expresiones de potencia instantánea en términos sinusoidales puros tiende a volverse más 
extenso a medida que las tensiones y corrientes instantáneas son diferentes, por lo que la deducción completa y detallada de la expresión de potencia instantánea no es incluida por falta de espacio en el artículo. Teniendo en cuenta un armónico de tensión ( $\left.\mathrm{v}_{\mathrm{zm}}\right)$ y un armónico de corriente (i $\left.i_{z n}\right)$ en la ecuación (24) se muestra la potencia instantánea por fase, en la ecuación (25) se muestra la potencia instantánea trifásica:

$$
\begin{aligned}
& \mathrm{p}_{z m 1}(t)=v_{z m} i_{z 1}=\left.v_{z m}\right|_{z 1}\left\{\begin{array}{l}
\cos \left[(n-m)\left(\omega_{1} t-\varphi_{z}\right)+\beta_{z 1}-\alpha_{z m}\right]- \\
\cos \left[(n+m)\left(\omega_{1} t-\varphi_{z}\right)+\beta_{z}+\alpha_{z m}\right]
\end{array}\right\} \\
& p_{m n}(t)=\sum_{z=-a, b, c} V_{z m} I_{z n}\left\{\begin{array}{l}
\cos \left[(n-m)\left(\omega_{1} t-\varphi_{z}\right)+\beta_{z z}-\alpha_{z m}\right]- \\
\cos \left[(n+m)\left(\omega_{1} t-\varphi_{z}\right)+\beta_{z z}+\alpha_{z m}\right]
\end{array}\right\}
\end{aligned}
$$

Observe que esta expresión de potencia está compuesta por dos términos cosenoidales puros de frecuencias angulares $(n-m) \omega_{1}$ y $(n+m) \omega_{1}$, en los que la secuencia de rotación tiene que ver con los términos $(\mathrm{n}-\mathrm{m}) \varphi_{\mathrm{z}}$ y $-(\mathrm{n}+\mathrm{m}) \varphi_{\mathrm{z}}$. La expresión anterior, de forma general, verifica el estudio realizado en la sección 5 de Emanuel (1993b) para la fase $b$. La potencia provocada por cualquier componente de la tensión y de la corriente puede cuantificarse de la siguiente manera:

$\mathrm{S}_{\mathrm{eN}}=3 \mathrm{~V}_{\mathrm{m}} \mathrm{I}_{\mathrm{n}}$

\section{MEDIDAS EXPERIMENTALES}

En esta sección se muestran los resultados experimentales obtenidos mediante la implementación en el laboratorio, la carga que contiene los fenómenos de desfase, distorsión y desequilibrio (figura 1). El desfase fue implementado con ayuda de tres bobinas de $206 \mathrm{mH}$ cada una, la distorsión fue implementada con un rectificador con carga $R / / C(R=60 \Omega, C=250 \mu f)$, y el desequilibrio fue implementado por la conexión de una única resistencia en la fase a de $242 \Omega$.

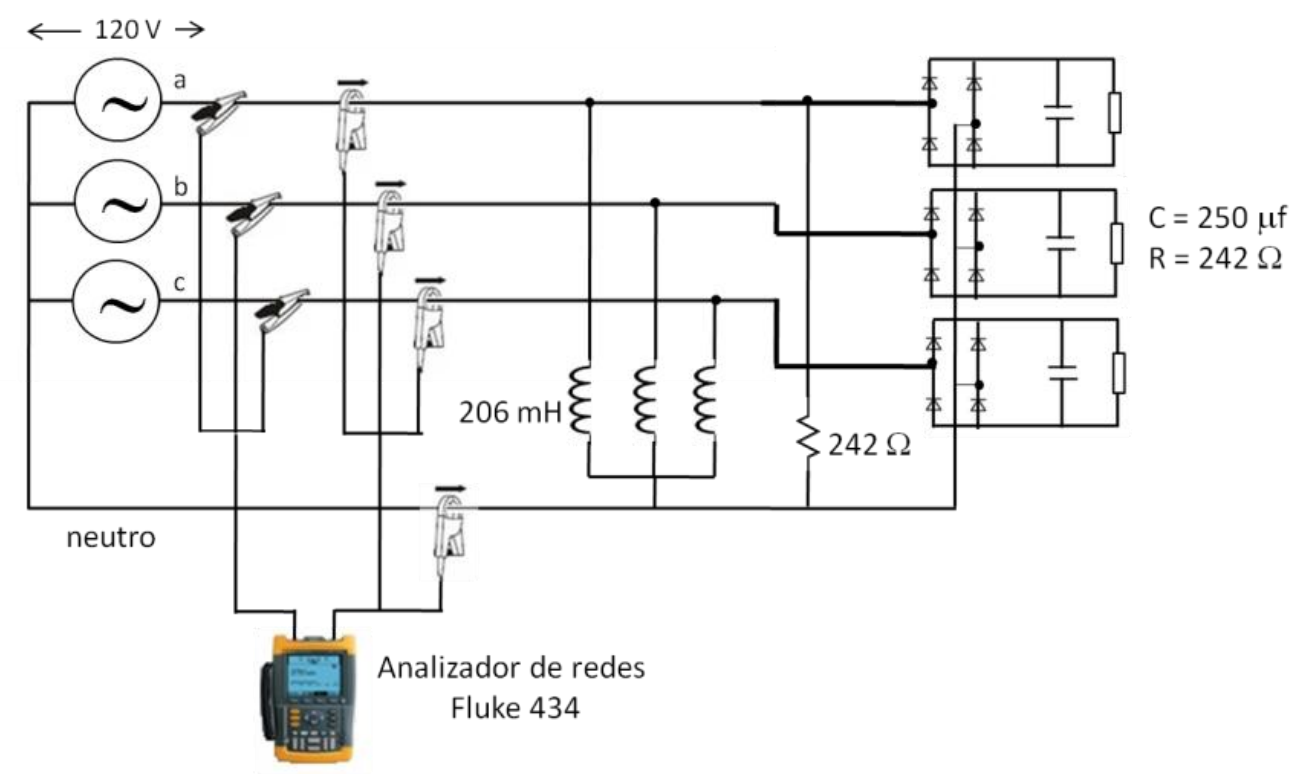

Fig 1. Montaje en el laboratorio.

Para la medida de las tensiones y las corrientes fue utilizado el analizador de redes Fluke 434. La tensión de alimentación está levemente distorsionada y se encuentra prácticamente equilibrada, la corriente de la carga contiene los fenómenos de desfase, distorsión, y desequilibrio, las formas de onda pueden verse en la figura 2. 
A partir de las medidas de voltaje y corriente fueron obtenidas algunas potencias instantáneas que servirán para validar las ecuaciones de la sección anterior y explicar los flujos de potencia de los sistemas eléctricos. En la figura 3 se muestran los flujos de potencia causados por componentes resistivos e inductivos equilibrados en la carga.

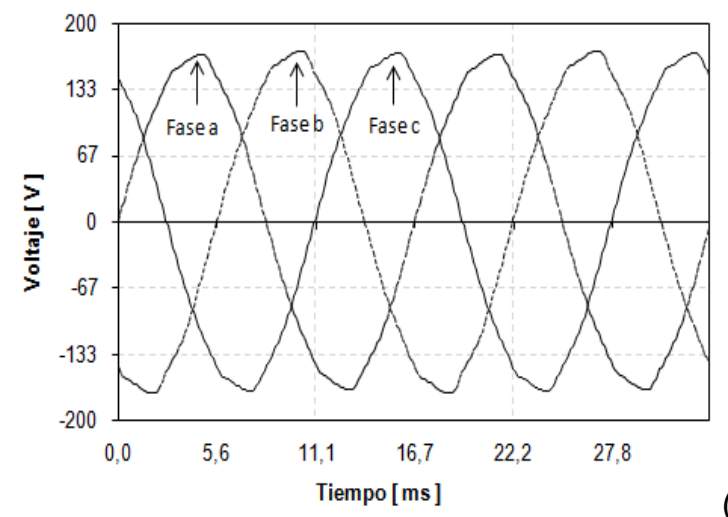

(a)

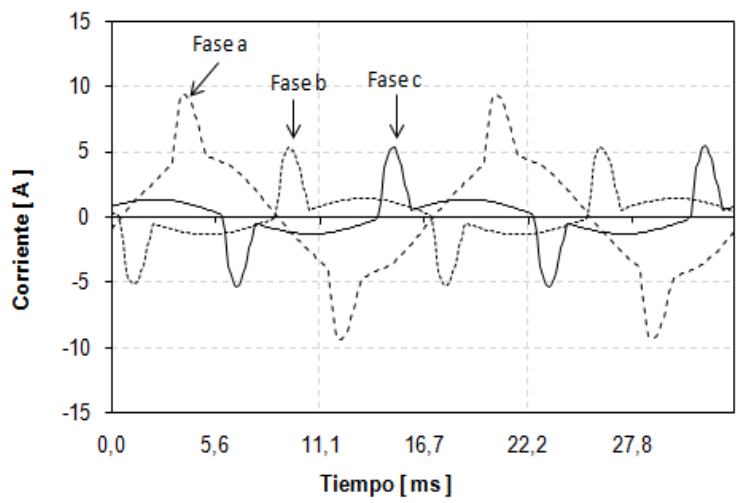

(b)

Fig 2. (a) Forma de onda de tensión. (b) Forma de onda de corriente de la carga.

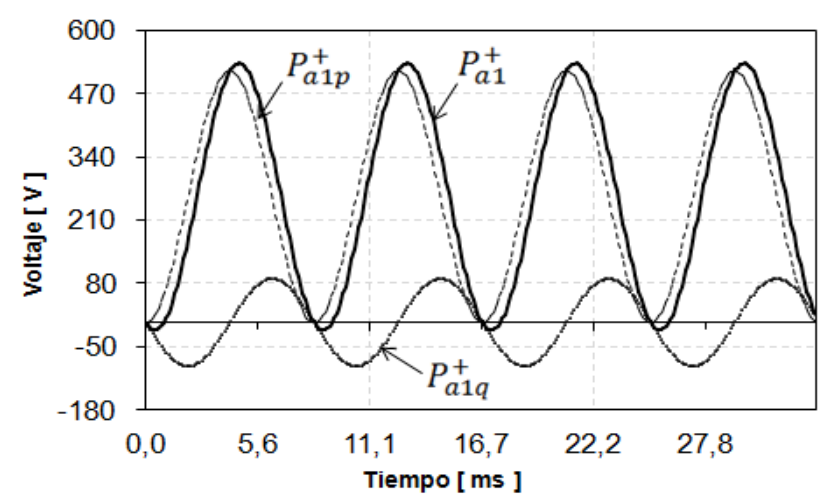

(a)

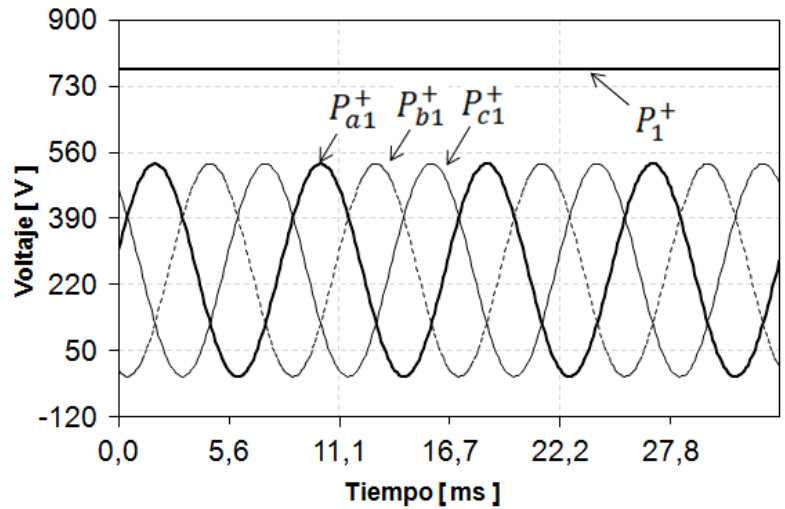

(b)

Fig 3. Potencias fundamentales equilibradas. (a) fase $a$. (b) fases $a, b$, c y potencia trifásica.

En la figura 3 (a) se muestra el flujo de potencia fundamental de secuencia positiva ( $\mathrm{P}_{\mathrm{a} 1}^{+}$) de la fase $a$ con sus respectivos flujos de potencia activa ( $\left.\mathrm{P}_{\mathrm{a} 1 \mathrm{p}}^{+}\right)$y reactiva $\left(\mathrm{P}_{\mathrm{a1q}}^{+}\right)$. $\mathrm{P}_{\mathrm{a} 1 \mathrm{p}}^{+}$es un flujo de potencia de valor medio positivo representando la transferencia de potencia útil desde la fuente hacia la carga. $\mathrm{P}_{\mathrm{a} 1 \mathrm{q}}^{+}$es un flujo de valor medio cero por lo que representa un intercambio bidireccional de potencia entre la fuente de potencia y la carga. En la figura 3 (b) se muestra el flujo de potencia fundamental de secuencia positiva de las fases $a, b, y c\left(\mathrm{p}_{\mathrm{a} 1}^{+}, \mathrm{p}_{\mathrm{b} 1}^{+}, \mathrm{p}_{\mathrm{c} 1}^{+}\right)$y la potencia trifásica $\left(\mathrm{p}_{1}^{+}\right)$. Los flujos de potencia $\mathrm{p}_{\mathrm{a} 1}^{+}, \mathrm{p}_{\mathrm{b} 1}^{+}, \mathrm{p}_{\mathrm{c} 1}^{+}$están compuestos por sus respectivas componentes de potencia activa y reactiva, estas potencias al sumarse sólo contienen componentes activas de potencia $\left(\mathrm{p}_{1}^{+}\right)$, por lo que $\mathrm{p}_{1}^{+}$sólo esta compuesta por componentes de potencia activa y su valor es positivo representando la transferencia útil de potencia desde la fuente hacia la carga. Las componentes de potencia reactiva se anulan en la carga de tal manera que no representan transferencia útil de potencia. En la figura 4 se muestran los flujos de potencia debido al desequilibrio de la carga y la asimetría de la red (carga resistiva en la fase a). 


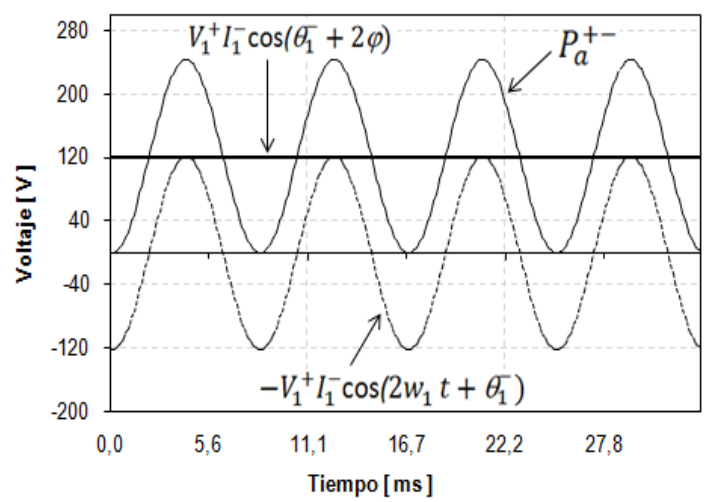

(a)

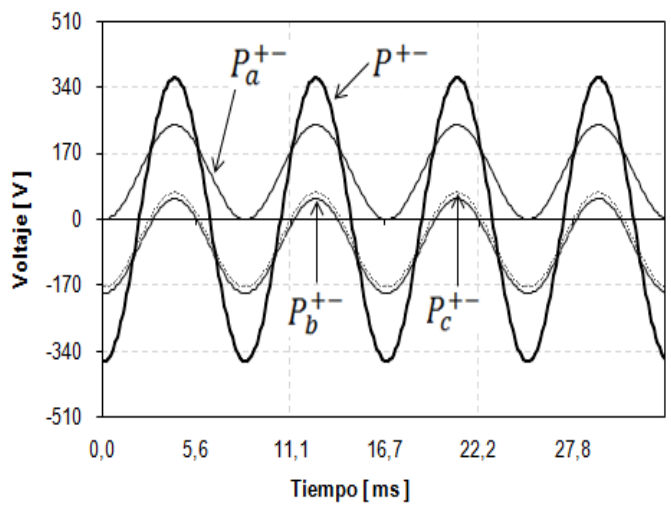

(b)

Fig 4. Potencias fundamentales desequilibradas. (a) fase $a$. (b) fases $a, b, c$ y potencia trifásica.

En la figura 4 (a) puede verse el flujo de potencia de desequilibrio fundamental de la fase a causado por corrientes fundamentales de secuencia negativa ( $\mathrm{p}_{\mathrm{a}}^{ \pm}$), $\mathrm{p}_{\mathrm{a}}^{ \pm}$esta compuesta por un flujo de valor medio positivo y un flujo de valor medio nulo. En la figura 4 (b) pueden verse los flujos de potencia causados por el desequilibrio de la carga de las fases $a, b$ y $c$, su suma es el flujo trifásico $\left(\mathrm{p}^{ \pm}\right), \mathrm{p}^{ \pm}$es de valor medio nulo por lo que no representa la transferencia útil de potencia a pesar de estar conformado por potencias de valor medio no nulo por fase. En la figura 5 se muestran los flujos de potencia causados por los componentes no lineales de la carga (rectificador).

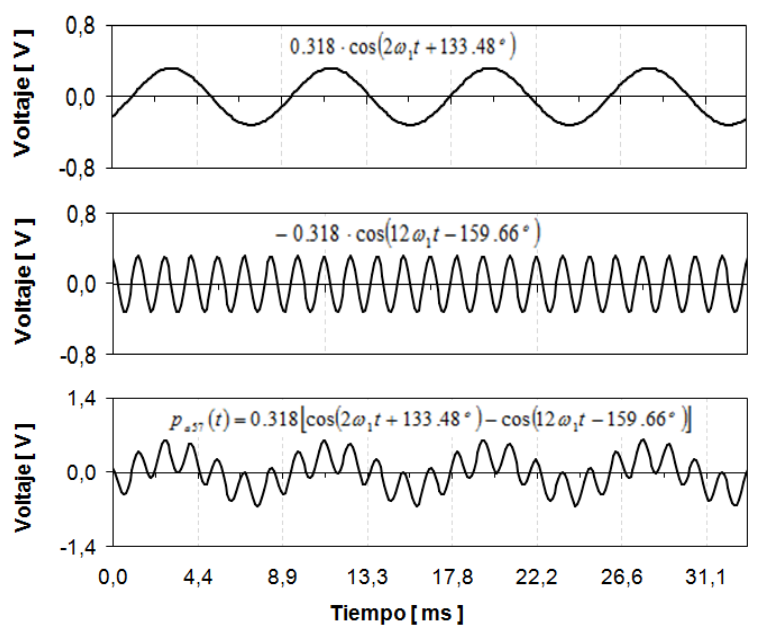

(a)

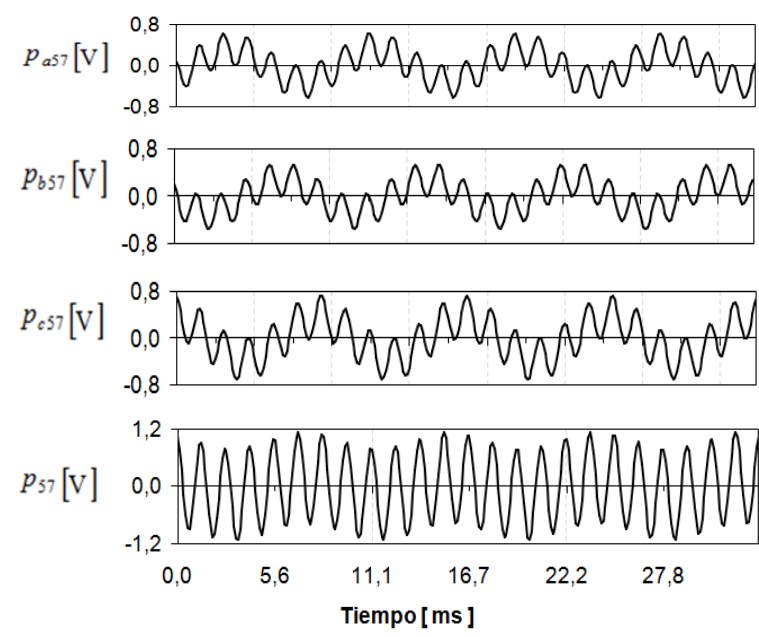

(b)

Fig 5. Potencias no fundamentales. (a) fase $a$. (b) fases $a, b, c$ y potencia trifásica.

En la figura 5 (a) puede verse el flujo de potencia no fundamental de la fase a causado por el quinto armónico de tensión y el séptimo armónico de corriente, este flujo de potencia de valor medio nulo está compuesto por dos flujos de potencia de valor medio nulo. En la figura 5 (b) pueden verse los flujos de potencia de las fases $a, b$, y $c\left(\mathrm{p}_{\mathrm{a} 57}, \mathrm{p}_{\mathrm{b} 57}, \mathrm{p}_{\mathrm{c57}}\right)$, su suma es el flujo trifásico ( $\left.\mathrm{P}_{57}\right), \mathrm{p}_{57}$ es de valor medio nulo por lo que no representa transferencia útil de potencia desde la fuente de potencia hacia la carga. El único flujo de potencia que representa una transferencia efectiva de potencia desde la fuente de potencia hacia la carga es $\mathrm{p}_{\mathrm{a} 1 \mathrm{p}}^{+}$, los otros flujos de potencia son de valor medio nulo o se anulan en la carga y no existe transferencia de potencia. Para cuantificar los flujos de potencia se utilizan las magnitudes de potencia definidas en la IEEE Std 1459 (2010) las cuales se encuentran resumidas en la tabla 1. 
Tabla 1: Cuantificación de los flujos de potencia mediante la IEEE Std. 1459 (2010).

\begin{tabular}{|c|c|c|c|}
\hline \multicolumn{4}{|c|}{$\mathrm{S}_{\mathrm{z}}=1383.1 \mathrm{vA}$} \\
\hline \multicolumn{3}{|c|}{$s_{\mathrm{s} 1}=1145.6 \mathrm{VA}$} & \multirow{3}{*}{$S_{\mathrm{zW}}=775.04 \mathrm{VA}$} \\
\hline & $5 v A$ & \multirow{2}{*}{$S_{\mathrm{v} 1}=799.4 \mathrm{VA}$} & \\
\hline $\mathrm{P}_{1}^{+}=775 \mathrm{~W}$ & $\mathrm{Q}_{1}^{+}=269.5 \mathrm{war}$ & & \\
\hline
\end{tabular}

La potencia $S_{e}$ cuantifica el intercambio de potencia entre la fuente de potencia y la carga está compuesta por la totalidad de los flujos de potencia que fluyen por el sistema eléctrico, $S_{e}$ se divide en $\mathrm{S}_{\mathrm{e} 1}$ y $\mathrm{S}_{\mathrm{eN}}$. La potencia $\mathrm{S}_{\mathrm{e} 1}$ cuantifica todos los flujos de potencia debido a componentes fundamentales de corriente y de tensión, mientras que $\mathrm{S}_{\mathrm{eN}}$ cuantifica todos los flujos de potencia causado por componentes no fundamentales de corriente y de tensión y es un flujo de potencia ineficiente de valor medio nulo. $S_{e 1}$ se divide en $S_{1}^{+}$y $S_{U 1}$, la potencia $S_{1}^{+}$cuantifica los flujos de potencia debido a componentes fundamentales y equilibradas de corriente y de tensión y contiene los flujos $\mathrm{P}_{1}^{+}$y $\mathrm{Q}_{1}^{+}$. La potencia $\mathrm{P}_{1}^{+}$corresponde a la potencia activa fundamental de secuencia positiva del sistema representando la transferencia útil de potencia, mientras que $\mathrm{Q}_{1}^{+}$es la potencia reactiva fundamental de secuencia positiva y representa un flujo de potencia bidireccional (ineficiente) que no representa transferencia útil de potencia. La potencia $\mathrm{S}_{\mathrm{U1}}$ cuantifica la potencia de desequilibrio fundamental que se anula en la carga de tal forma que no representa transferencia útil de potencia.

\section{CONCLUSIONES}

Se ha desarrollado la teoría de la potencia instantánea como extensión de los desarrollos previos existentes y como alternativa para cuantificar correctamente los fenómenos de potencia de los sistemas eléctricos. Se ha demostrado mediante el estudio de los flujos de potencia instantánea que la única potencia que representa una transferencia eficiente de energía desde la red de suministro hacia la carga es $\mathrm{P}_{1}^{+}$. El resto de potencias instantáneas presentes en el sistema eléctrico son ineficientes ya que son potencias instantáneas bidireccionales o nulas que circulan entre la red de suministro y la carga. Estas potencias instantáneas no representan un intercambio efectivo de energía, provocando pérdidas y efectos nocivos en los sistemas eléctricos.

Se ha deducido una expresión de potencia instantánea (ecuación (24)) válida para sistemas eléctricos con tensiones asimétricas no fundamentales y con cargas no lineales desequilibradas. Con ayuda de esta expresión se puede cuantificar mejor la potencia de los sistemas eléctricos y entender con claridad los fenómenos que suceden en los sistemas eléctricos. Con la ecuación (24) es posible entender como fluye la potencia de los sistemas eléctricos y puede usarse en el diseño de compensadores o filtros activos para la mejora de la eficiencia energética como también en la detección de las fuentes de distorsión armónica, en la evaluación de la calidad de la potencia, y en la reglamentación de la facturación del consumo de la energía eléctrica.

La cuantificación de potencia mediante el uso de la teoría de la potencia instantánea coincide con la cuantificación de potencia realizada mediante la IEEE Std. 1459 (2010).

\section{AGRADECIMIENTOS}

Los autores agradecen a la UdeA por el apoyo de "Sostenibilidad 2009-2010".

\section{REFERENCIAS}

Akagi, H., S. Ogasawara, y H. Kim, The Theory of Instantaneous Power in Three-Phase Four-Wire Systems: A Comprehensive Approach, Conf Rec of IEEE IAC, 431-439, (1999). 
Chen, C., y Y. Hsu, A novel approach to the design of a shunt active filter for an unbalanced threephase four-wire system under nonsinusoidal conditions, IEEE Trans. Power Delivery, 15 (4), 12581264, (2000).

Czarnecki, L.S., Orthogonal decomposition of the currents in a 3-phase nonlinear asymmetrical circuit with a nonsinusoidal voltage source, IEEE Trans. Instrumentation and Measurement, 37 (1), 30-34, (1988).

Depenbrock, M., The FBD-Method, a Generally Applicable Tool for Analyzing Power Relations, IEEE Trans. Power Systems, 8 (2), 381-397, (1993).

Emanuel, A.E., Powers in nonsinusoidal situation: a review of definitions and physical meaning, IEEE Trans. Power Delivery, 5 (3), 1377-1389, (1990).

Emanuel, A.E., On the definition of power factor and apparent power in unbalanced polyphase circuits with sinusoidal voltage and currents, IEEE Trans. Power Delivery, 8 (3), 841-847, (1993a).

Emanuel, A.E., Apparent and reactive powers in three-phase systems: in search of a physical meaning and a better resolution, European Trans. Electrical Power, 3 (1), 7-14, (1993b).

Emanuel, A.E., Summary of IEEE Standard 1459: Definitions for the Measurement of Electric Power Quantities Ander Sinusoidal, Nonsinusoidal, Balanced, or Unbalanced Conditions, IEEE Trans. Industry Applications, 40 (3), 869-874, (2004).

Ferrero, A., y G. Superti-Furga, A new approach to the definition of power components in threephase systems under nonsinusoidal conditions, IEEE Trans. Instrumentation and Measurement, 40(3), pp 568-577 (1991).

Giadrossi, G., R. Menis, G. Sulligoi, y A. Tessarolo, Voltage stability analysis of all-electric cruise liners, SPEEDAM International Symposium on, 586-590, (2008).

Kim, H., F. Blaabjert, y B. Bak-Jensen, Spectral Analysis of Instantaneous Powers in Single-Phase and Three-Phase Systems with Use of p-qr Theory, IEEE Trans. Power Electronics, 17 (5), 711 720, (2002).

Orts, S., A. Abellán, F. Gimeno, S. Seguí, y J. Alfonso, Improved Shunt Active Power Compensator for IEEE Standard 1459 Compliance, IEEE Trans. Power Delivery, 25 (4), 26922701, (2010).

Pigazo, A., y V.M. Moreno, Accurate and Computationally Efficient Implementation of the IEEE 1459-2000 Standard in Three-Phase Three-Wire Power Systems, Power Delivery, IEEE Transactions on, 22 (1), 752-757, (2007).

Salmerón, P., y J. Montaño, Instantaneous Power Components in Polyphase Systems Under Nonsinusoidal Conditions, IEE Proc.-Sci. Meas. Tech., 143 (2), (1996).

Willems, J.L., J.A. Ghijselen, y A.E. Emanuel, The Apparent Power Concept and the IEEE Standard 1459-2000, IEEE Trans. Power Delivery, 20 (2), 876-884, (2005). 
